\title{
ON THE COEFFICIENTS OF VARIATION OF UPTIME AND DOWNTIME IN MANUFACTURING EQUIPMENT
}

\author{
JINGSHAN LI AND SEMYON M. MEERKOV
}

Received 28 November 2004

It was reported in the literature that the coefficients of variation of uptime and downtime of manufacturing equipment are often less than 1 . This technical paper is intended to provide an analytical explanation of this phenomenon. Specifically, it shows that the distributions of uptime and downtime have the coefficients of variation less than 1 if the breakdown and repair rates are increasing functions of time.

\section{Introduction}

The coefficients of variation of uptime and downtime $\left(\mathrm{CV}_{\text {up }}\right.$ and $\left.\mathrm{CV}_{\text {down }}\right)$ of the equipment on the factory floor have a significant effect on the design of lean production systems. Indeed, as has been shown in [2], lean buffering (i.e., the buffering necessary and sufficient to achieve a desired production rate of a manufacturing system) is an increasing function of $\mathrm{CV}_{\text {up }}$ and $\mathrm{CV}_{\text {down }}$, and therefore smaller $\mathrm{CV}$ s lead to leaner systems.

In designing lean systems, it is often assumed that the uptime and downtime $\left(t_{\text {up }}\right.$ and $\left.t_{\text {down }}\right)$ are distributed exponentially and, thus, $\mathrm{CV}_{\text {up }}=\mathrm{CV}_{\text {down }}=1$. Since in reality $t_{\text {up }}$ and $t_{\text {down }}$ may not be exponential, it is important to determine if lean buffering, based on the exponential assumption, overestimates or underestimates the real lean buffering. If $\mathrm{CV}_{\text {up }}$ and $\mathrm{CV}_{\text {down }}$ are less than 1, the lean buffers, designed using the exponential assumption, are too large; otherwise, they are too small.

The empirical evidence [3] indicates that $\mathrm{CV}_{\text {up }}$ is very often less than 1 ; this is also true for $\mathrm{CV}_{\text {down }}$ although to a lesser extent. As follows from the above, this is an important fact since it ensures that lean buffering, designed using the exponential assumption, will ensure the desired throughput (however, without the maximum leanness).

A question arises: what are "natural" causes which lead to $\mathrm{CV}_{\text {up }}$ and $\mathrm{CV}_{\text {down }}$ being less than 1? The purpose of this technical paper is to discuss this question and, thereby, characterize practical situations that lead to small $\mathrm{CV}_{\text {up }}$ and $\mathrm{CV}_{\text {down }}$.

To accomplish this, Section 2 describes the model of machines under consideration, Section 3 discusses a special case, in Section 4 a general scenario is considered, and in Section 5 conclusions are formulated. 


\section{Coefficients of variation of uptime and downtime}

\section{Model}

We consider machines, which could be in two states, up and down. When up, parts are produced; when down, no production takes place and the machine is under repair. Transition rates from up to down and from down to up are $p(t)$ and $r(t)$, respectively, where $t$ is the time the machine spent in the respective state. In other words, if the machine went up at $t=0$, it goes down during the infinitesimal interval $(t, t+\delta t)$ with probability $p(t) \delta t$. Similarly, transition from down to up occurs during $(t, t+\delta t)$ with probability $r(t) \delta t$.

Obviously, the uptime and downtime defined by this model are random variables with probability density functions (pdf's) induced by $p(t)$ and $r(t)$. If both $p(t)$ and $r(t)$ are constant, that is, $p(t)=p_{0}$ and $r(t)=r_{0}, t \geq 0$, the resulting random variables are exponential, and $\mathrm{CV}_{\text {up }}=\mathrm{CV}_{\text {down }}=1$. What happens when $p(t)$ and $r(t)$ are not constant is discussed next.

\section{Special case}

We analyze below the pdf and the coefficient or variation of the uptime; the downtime is treated similarly.

Assume that $p(t)$ is given by

$$
p(t)=p_{0} t^{a}, \quad p_{0}=\text { const }, a>-1, t \geq 0 .
$$

This implies that the breakdown rate is strictly monotonically increasing in time when $a>0$, constant when $a=0$ (the exponential case), and strictly monotonically decreasing when $-1<a<0$. The values of $a<-1$ are not included since they do not lead to valid pdf's (in the sense that the integrals of these functions are not finite).

The pdf and the CV of the uptime, induced by assumption (3.1), can be calculated as follows.

By the total probability formula, the probability that the machine is up at time $t+\delta t$ is given by

$$
P[\alpha(t+\delta t)=1]=P[\alpha(t+\delta t)=1 \mid \alpha(t)=1] P[\alpha(t)=1]
$$

where $\alpha(t)$ represents the state of the machine, that is,

$$
\alpha(t)= \begin{cases}1 & \text { if the machine is up at time } t \\ 0 & \text { if the machine is down at time } t\end{cases}
$$

As follows from the machine model of Section 2 and (3.1), the conditional probability that the machine is up at $t+\delta t$, given that it is up at $t$, is

$$
P[\alpha(t+\delta t)=1 \mid \alpha(t)=1]=1-p_{0} t^{a} \delta t .
$$


Substituting this into (3.2) and rearranging terms, we obtain

$$
\frac{P[\alpha(t+\delta t)=1]-P[\alpha(t)=1]}{\delta t}=-p_{0} t^{a} P[\alpha(t)=1]
$$

When $\delta t \rightarrow 0$, this becomes a first-order linear differential equation

$$
\frac{d P[\alpha(t)=1]}{d t}=-p_{0} t^{a} P[\alpha(t)=1]
$$

with initial condition

$$
P[\alpha(0)=1]=1 .
$$

Solving this initial value problem, we obtain the probability that the machine is up at time $t$ assuming that it was up at time 0

$$
P[\alpha(t)=1]=e^{-\int_{0}^{t} p_{0} t^{a} d t}=e^{-p_{0} t^{a+1} /(a+1)}
$$

To determine the pdf of $t_{\mathrm{up}}$, we calculate the joint probability that the machine is up at time $t$ and down at time $t+\delta t$. Using the formula for the probability of the intersection of two events and (3.8), we obtain

$$
\begin{aligned}
& P[\{\alpha(t)=1\} \cap\{\alpha(t+\delta t)=0\}] \\
& \quad=P[\alpha(t+\delta t)=0 \mid \alpha(t)=1] P[\alpha(t)=1] \\
& \quad=\left(p_{0} t^{a} \delta t\right)\left(e^{-p_{0} t^{a+1} /(a+1)}\right) \\
& \quad=p_{0} t^{a} e^{-p_{0} t^{a+1 /(a+1)}} \delta t .
\end{aligned}
$$

This implies that the pdf of $t_{\mathrm{up}}$ is

$$
f_{t_{\mathrm{up}}}(t)=p_{0} t^{a} e^{-\left(p_{0} /(a+1) t^{a+1}\right.}, \quad a>-1, t \geq 0
$$

To verify that this is a valid pdf, we integrate it over $[0, \infty)$. Using the substitution $x=t^{a+1}$, we obtain

$$
\begin{aligned}
\int_{0}^{\infty} f_{t_{\text {up }}}(t) d t & =\int_{0}^{\infty} p_{0} t^{a} e^{-\left(p_{0} /(a+1)\right) t^{a+1}} d t \\
& =\int_{0}^{\infty} \frac{p_{0}}{a+1} e^{-\left(p_{0} /(a+1)\right) x} d x=1 .
\end{aligned}
$$


4 Coefficients of variation of uptime and downtime

The expected value and the variance of distribution (3.10) can be expressed as follows:

$$
\begin{aligned}
E\left[t_{\mathrm{up}}\right]= & \int_{0}^{\infty} t p_{0} t^{a} e^{-\left(p_{0} /(a+1) t^{a+1}\right.} d t \\
= & {\left.\left[-t e^{-\left(p_{0} /(a+1)\right) t^{a+1}}-\left(\frac{p_{0}}{a+1} t^{a+1}\right)^{-1 /(a+1)} \Gamma\left(\frac{1}{a+1}, \frac{p_{0}}{a+1} t^{a+1}\right)\right]\right|_{0} ^{\infty} } \\
= & \frac{1}{a+1}\left(\frac{a+1}{p_{0}}\right)^{1 /(a+1)} \Gamma\left(\frac{1}{a+1}\right), \\
\operatorname{Var}\left[t_{\mathrm{up}}\right]= & \int_{0}^{\infty} t^{2} p_{0} t^{a} e^{-\left(p_{0} /(a+1)\right) t^{a+1}} d t-\left(E\left[t_{\mathrm{up}}\right]\right)^{2} \\
= & {\left.\left[-t^{2} e^{-\left(p_{0} /(a+1) t^{a+1}\right.}-\frac{2}{a+1}\left(\frac{p_{0}}{a+1}\right)^{-2 /(a+1)} \Gamma\left(\frac{2}{a+1}, \frac{p_{0}}{a+1} t^{a+1}\right)\right]\right|_{0} ^{\infty} } \\
& -\frac{1}{(a+1)^{2}}\left(\frac{a+1}{p_{0}}\right)^{2 /(a+1)}\left[\Gamma\left(\frac{1}{a+1}\right)\right]^{2} \\
= & \frac{2}{a+1}\left(\frac{a+1}{p_{0}}\right)^{2 /(a+1)} \Gamma\left(\frac{2}{a+1}\right)-\frac{1}{(a+1)^{2}}\left(\frac{a+1}{p_{0}}\right)^{2 /(a+1)}\left[\Gamma\left(\frac{1}{a+1}\right)\right]^{2},
\end{aligned}
$$

where $\Gamma(\cdot)$ is the gamma function defined by

$$
\Gamma(x)=\int_{0}^{\infty} t^{x-1} e^{-t} d t
$$

Thus, the coefficient of variation is

$$
\mathrm{CV}_{\text {up }}=\left[\frac{2(a+1) \Gamma(2 /(a+1))}{[\Gamma(1 /(a+1))]^{2}}-1\right]^{1 / 2} \text {. }
$$

The values of $\mathrm{CV}_{\text {up }}$ for various $a$ 's are given in Table 3.1. Clearly, positive $a$ 's, that is, increasing breakdown rates, lead to $\mathrm{CV}_{\text {up }}<1$, while decreasing breakdown rates result in $\mathrm{CV}_{\text {up }}$ greater than 1. Thus, increasing (resp., decreasing) breakdown rates are responsible for having $\mathrm{CV}_{\text {up }}<1$ (resp., $\mathrm{CV}_{\text {up }}>1$ ).

Remark 3.1. Note that when $a=1$, that is, the breakdown rate is linearly increasing in time, the pdf of $t_{\text {up }}$ (3.10) reduces to the well-known Rayleigh pdf. Also note that for any $a>-1$, pdf (3.10) is similar to the Weibull distribution widely used in reliability theory.

\section{General case}

Consider a machine with breakdown rate satisfying the following inequality:

$$
p\left(t_{1}\right)<p\left(t_{2}\right), \quad 0 \leq t_{1}<t_{2}<\infty
$$

Unlike (3.1), this assumption implies a general monotonic growth of the breakdown rate on the infinite time interval. Machines satisfying (4.1) may be referred to as aging on the infinite time interval. Without exploring particular pdf's induced by (4.1), it has been 
Table 3.1. $\mathrm{CV}_{\mathrm{up}}$ as a function of $a$.

(a) $a>0$.

\begin{tabular}{c|ccccccccc}
\hline$a$ & 0.5 & 1 & 1.5 & 2 & 2.5 & 3 & 5 & 10 & 100 \\
\hline $\mathrm{CV}_{\text {up }}$ & 0.6790 & 0.5227 & 0.4279 & 0.3634 & 0.3165 & 0.2805 & 0.1938 & 0.1099 & 0.0126 \\
\hline
\end{tabular}

(b) $-1<a<0$.

\begin{tabular}{c|cccccccc}
\hline$a$ & -0.05 & -0.1 & -0.15 & -0.25 & -0.5 & -0.75 & -0.9 & -0.98 \\
\hline $\mathrm{CV}_{\text {up }}$ & 1.0530 & 1.1130 & 1.1815 & 1.3529 & 2.2361 & 8.3066 & 429.8314 & $3.1763 E+14$ \\
\hline
\end{tabular}

shown in [1] that the uptime of such machines has the following property:

$$
\operatorname{Var}\left[t_{\mathrm{up}}\right]<\left(E\left[t_{\mathrm{up}}\right]\right)^{2}
$$

This implies that

$$
\mathrm{CV}_{\text {up }}<1 .
$$

Thus, the conclusion of Section 3 also holds for a more general type of monotonically increasing breakdown rates.

In reality, however, when preventative maintenance (PM) programs are implemented, assumption (4.1) might not take place on the infinite time interval but only between each pair of successive PM operations, that is,

$$
p\left(t_{1}\right)<p\left(t_{2}\right), \quad \text { for } T i \leq t_{1}<t_{2} \leq T(i+1), i=0,1,2, \ldots,
$$

where $T$ is the period of PM operations. Under this condition, (4.2) may or may not take place. Assume, however, that the PM period, $T$, is much larger than the expected value of $t_{\text {up }}$ between any two consecutive PM operations, that is,

$$
\frac{E\left[t_{\mathrm{up}} \mid T i \leq t \leq T(i+1)\right]}{T} \ll 1, \quad i=0,1,2, \ldots
$$

The machine satisfying (4.4), (4.5) can be called aging on large PM periods. When the ratio in the left-hand side of (4.5) is sufficiently small, inequality (4.2) can again be established.

Thus, a general reason for having $\mathrm{CV}_{\text {up }}<1$ is that the equipment is aging, either on the infinite time interval or on large PM periods.

\section{Conclusions}

In many situations, manufacturing equipment on the factory floor is aging in both casual and technical senses (3.1) with $a>0$, or (4.1), or (4.4), (4.5). Similarly, it is reasonable to assume that many repair operations (excluding PM and tool change downtime) are also 
"aging" in the sense that either

$$
r(t)=r_{0} t^{a} \delta t, \quad r_{0}=\text { const, } a>0, t \geq 0,
$$

or, more generally,

$$
r\left(t_{1}\right)<r\left(t_{2}\right), \quad 0 \leq t_{1}<t_{2}<\infty .
$$

In all these cases, as the above analysis shows,

$$
\mathrm{CV}_{\text {up }}<1, \quad \mathrm{CV}_{\text {down }}<1 .
$$

On the other hand, if $p(t)$ and $r(t)$ are decreasing in time, the resulting CVs are greater than 1 . Thus, increasing transition rates appear to be the reason for the empirical evidence reported in [3].

\section{References}

[1] R. E. Barlow, F. Proschan, and L. C. Hunter, Mathematical Theory of Reliability, John Wiley \& Sons, New York, 1965.

[2] E. Enginarlar, J. Li, S. M. Meerkov, and R. Q. Zhang, Buffer capacity for accommodating machine downtime in serial production lines, Int. J. Prod. Res. 40 (2002), no. 3, 601-624.

[3] R. R. Inman, Empirical evaluation of exponential and independence assumptions in queueing models of manufacturing systems, Prod. Oper. Manag. 8 (1999), 409-432.

Jingshan Li: Manufacturing Systems Research Laboratory, GM Research \& Development Center, Warren, MI 48090-9055, USA

E-mail address: jingshan.li@gm.com

Semyon M. Meerkov: Department of Electrical Engineering and Computer Science, University of Michigan, Ann Arbor, MI 48109-2122, USA

E-mail address: smm@eecs.umich.edu 


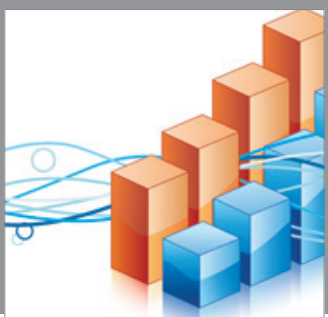

Advances in

Operations Research

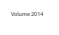

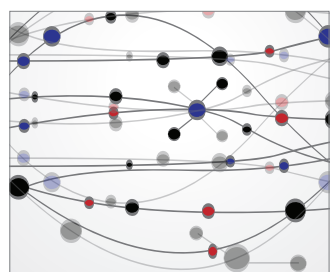

\section{The Scientific} World Journal
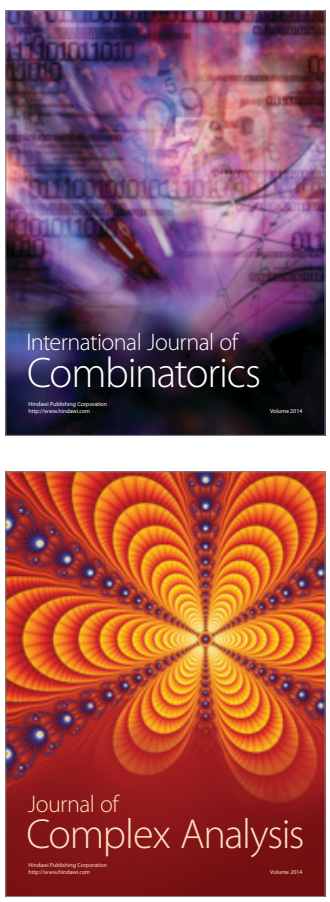

International Journal of

Mathematics and

Mathematical

Sciences
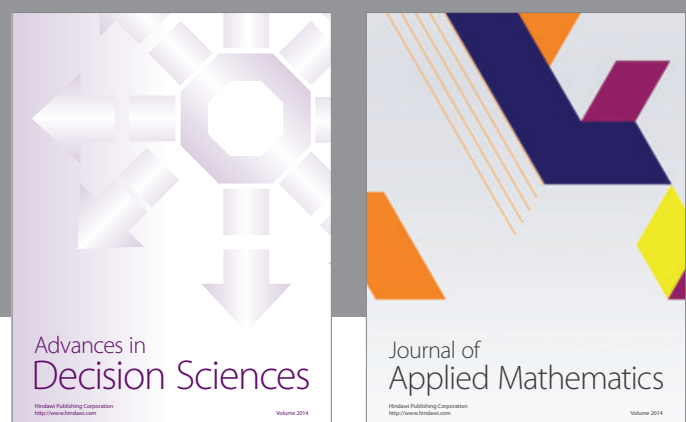

Journal of

Applied Mathematics
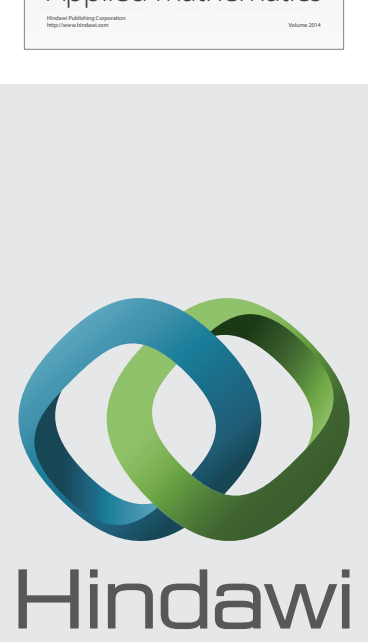

Submit your manuscripts at http://www.hindawi.com
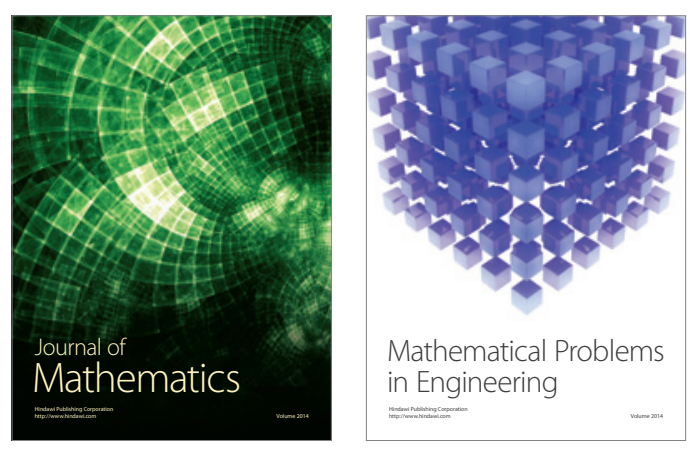

Mathematical Problems in Engineering
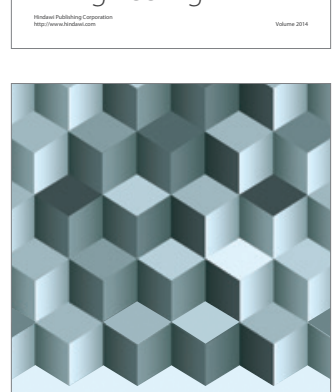

Journal of

Function Spaces
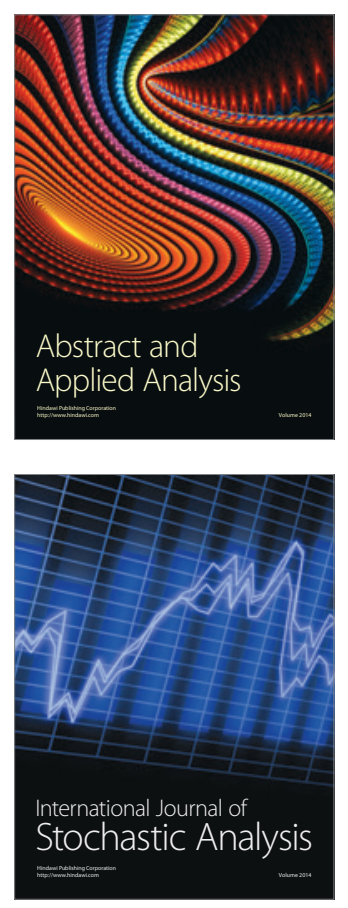

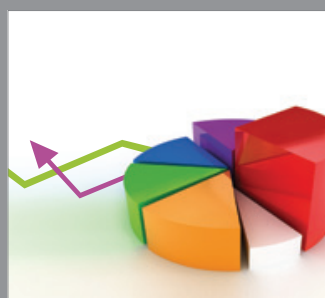

ournal of

Probability and Statistics

Promensencen
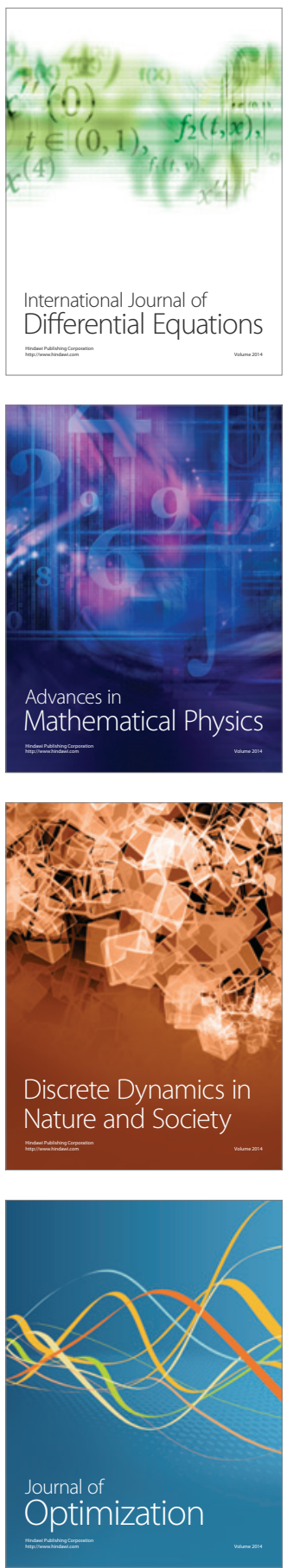